\title{
A História da Psicologia e seu ensinamento há 40 anos desde "'Should the History of Science Be Rated $X$ ?" por Stephen Brush
}

\section{History of Psychology and it's Teaching at 40 years since 'Should the History of Science Be Rated X?' by Stephen Brush}

\section{La Historia de la Psicología y su Enseñanza a 40 años de 'Should the History of Science Be Rated X? de Stephen Brush}

\author{
Catriel Fierro \\ Facultad de Psicología - Universidad Nacional de Mar del Plata, Mar del Plata, \\ Buenos Aires, Argentina.
}

\begin{abstract}
RESUMO
Comemorando os quarenta anos do artigo Should the History of Science Be Rated $X$ ? de Stephen Brush, propõe-se uma reflexão a respeito da relação entre o ensino da história da psicologia, a historiografia e a formação dos psicólogos. Em primeiro lugar, sintetiza-se a argumentação de Brush em torno à história da ciência e seu ensino. Depois, descreve-se a apropriação desde a história da psicologia e seu ensino de certas problemáticas citadas pelo autor, detalhando a questão da raiz geracional do conhecimento histórico e a necessidade da ampliação do gênero e tarefa histórica. Finalmente, menciona-se a questão da tensão entre a vertente curricular e acadêmica da história. Conclui-se que a adoção de uma moderada atitude crítica e de um marco historiográfico sociológico por parte dos docentes conseguiria evitar um ensino da história parcial e enviesado.
\end{abstract}

Palavras-chave: história, psicologia, ensino, história whig, história social.

\begin{abstract}
Commemorating forty years since 'Should the History of Science Be Rated $X$ ?' by Stephen Brush, a reflection on the relation between the teaching of the History of psychology, historiography and the training of psychologists is proposed. Brush's argument about the history of science and its teaching is first summarized. The appropriation in the History of psychology and its teaching of certain problems alluded to by Brush are then described, detailing the issues about the generational roots of historical knowledge and the need to expand the genre of history and the historian's tasks. Finally, the issue about the tension between the curricular and the academic aspects of history is detailed. It is concluded that the adoption of a moderate critical attitude and a sociological historiographical framework by teachers could avoid a biased and partialized teaching of the history of psychology.
\end{abstract}

Keywords: history, psychology, teaching, whig history, social history.

\section{RESUMEN}


Conmemorando los cuarenta años del artículo Should the History of Science Be Rated $X$ ? de Stephen Brush, se propone una reflexión acerca de la relación entre la enseñanza de la historia de la psicología, la historiografía y la formación de los psicólogos. Se sintetiza primero la argumentación de Brush en torno a la historia de la ciencia y su enseñanza. Se describe luego la apropación desde la historia de la psicología y su enseñanza de ciertas problemáticas aludidas por el autor, detallándose la cuestión de la raíz generacional del conocimiento histórico y la necesidad de ampliación del género y tarea históricos. Finalmente, se detalla la cuestión de la tensión entre la vertiente curricular y la vertiente académica de la historia. Se concluye que la adopción de una moderada actitud crítica y de un marco historiográfico sociológico por parte de los docentes lograría evitar una enseñanza de la historia parcializada y sesgada.

Palabras clave: historia, psicología, enseñanza, historia whig, historia social.

\section{La Historia de la ciencia y la censura cegún Stephen Brush}

En 1974, en un número de la popular revista norteamericana Science, el físico e historiador Stephen Brush publicaba una apología a la historia de la ciencia como contenido en la formación de los científicos. El artículo, traducido al español como "¿Debería calificarse $X$ a la Historia de la Ciencia?", retomaba el sistema clasificatorio cinematográfico estadounidense como analogía para vetar, simbólicamente, la enseñanza de la historia de la ciencia del entrenamiento de los científicos en el ámbito de la educación media y superior. La razón, según Brush, era que "la forma en que los científicos se comportan (según los historiadores) podría no ser un buen modelo para los estudiantes" (Brush, 1974, pág. 1164).

A lo largo de las páginas del artículo, Brush describe -con cierto tono irónico, pero con argumentaciones sólidas y ejemplos de científicos revolucionarios- cómo la historia de la ciencia contemporánea a él (la 'nueva historia de la ciencia') habría cambiado radicalmente la forma de concebir a la actividad científica y a sus representantes. Brush admitía que el uso que se había dado hasta el momento a la historia de la ciencia como contenido curricular era, principalmente, adoctrinador -o pedagógico, o socializador-: la historia de la ciencia, especialmente como la reflejaban los clásicos manuales -tanto los manuales sistemáticos como los manuales propiamente históricosaparecía como una sucesión de descubridores, alternada con una sucesión de cambios teóricos, estos últimos los cuales se debían principalmente al refinamiento metodológico de la técnica experimental y a la acumulación de conocimientos.

Aunque clásicamente se sostuvo que la historia era fuente de ideas novedosas, fuente de actitudes juiciosas y fuente de comprensión del estado actual de la ciencia, Brush rastrea cómo los manuales e historiadores de la ciencia en realidad no consideran que dicha 
historia sea una parte esencial del currículo científico, sirviendo sólo a los fines de que el estudiante adquiera refinamiento metodológico. Aún más, sostiene Brush, algunos científicos ven en la enseñanza de la historia una pérdida de tiempo, dado que la historia sólo sería el depósito de los errores ya superados de científicos de otras épocas.

Sin embargo, el autor sostiene que con la llegada de los nuevos usos de la historia y con los nuevos planteamientos en filosofía de la ciencia -elementos que identifica principalmente con Thomas Kuhn-, las revisiones históricas no sólo reivindicarían el lugar del curso de grado de enfoque histórico: también demostrarían aspectos de los grandes científicos -figuras eminentes de la clásica revolución científica- y de la propia dinámica de la ciencia que difícilmente serían aquellos que los docentes de historia querrían transmitir a los alumnos. La nueva historia y sus perspectivas revisionistas irían en contra, necesariamente, de los usos adoctrinadores de la historia de la ciencia, en el sentido que "violentarían el ideal profesional y la imagen pública de los científicos en tanto investigadores abiertos y racionales, quienes procederían metódicamente, fundamentados incontrovertiblemente en los resultados de experimentos controlados, buscando objetivamente la verdad, sea cual sea el resultado" (Brush, 1974, pág. 1164).

El impacto del artículo de Brush fue considerable y reavivó la discusión en torno a las ventajas y desventajas de la enseñanza de la historia de la ciencia en el currículo universitario, la cual llega incluso hasta nuestros días (Gooday, Lynch, Wilson, \& Barsky, 2008). Contemporáneo a la publicación de dicho artículo, en el campo disciplinar de la historia de la psicología se hallaba en pleno desarrollo un proceso de cambio que había comenzado ya hacia 1960 en torno al Ilamamiento -realizado por algunos representantes del ámbito- de no permitir que el área histórica de la disciplina se deslizara hacia el olvido (Watson, 1960). Este proceso de cambio consistía, en su conjunto, en la emergencia de la denominada 'nueva historia' de la psicología (Furumoto, 1989): un marco historiográfico novedoso que permitió a la historia de la psicología dejar de ser sistematología o psicología contemporánea y acercarse al campo de estudio de la historia de la ciencia.

Dada la emergencia y configuración paralela de los nuevos enfoques historiográficos en historia de la ciencia (física) e historia de la psicología, y ante la aparición dentro de estos campos de la cuestión de los fines de la enseñanza universitaria de ambas disciplinas, hipotetizamos que es de utilidad para una historia de la historiografía de la psicología (Klappenbach, 2000) analizar la forma en que algunas de las cuestiones y problemáticas de índole teórica y pedagógica puestas en relieve por Brush fueron adoptadas y resueltas -o al menos enfrentadas- en el ámbito de la enseñanza de la historia de la psicología. Para tal fin se realiza una revisión 
bibliográfica sobre tópicos que en historia e historiografía de la psicología hayan recogido los planteos de Brush. Los planteos del autor son de especial importancia si consideramos que la propia tradición clásica de la historia de la psicología y sus reconstrucciones manualizadas -se ha sostenido- perseguían ideales legitimadores muy semejantes a los que Brush imputa a la clásica historia de la ciencia (O'Donnell, 1979). En este sentido es que la tradición clásica en historia de la psicología fue una tradición con intereses pedagógicos: constituida predominantemente con el afán de lograr la socialización de académicos e ingresantes a la psicología (Ash, 1983), incurriendo por esto en graves tergiversaciones e imprecisiones.

\section{La disciplina histórica: historia ampliada y raíces generacionales}

Según Brush, debe partirse de una definición ampliada de la historia de la ciencia. La historia no debe circunscribirse a enumerar teorías y grandes descubridores. Al contrario, debe ser (o aspirar a ser) un conjunto coherente de relatos sobre descubrimientos $y$, sobre propuestas teóricas. Además, debe incluir entre sus conocimientos las diversas interrelaciones entre la ciencia que es su objeto de estudio y las otras formas de actividades humanas (los aspectos societales, los aspectos metafísicos, los aspectos institucionales, entre otros). Brush reconocía, tal como lo hacía simultáneamente Walter Weimer (1974) acerca de la historiografía de la psicología, que "la historia no es meramente un registro estático de hechos, sino también las interpretaciones propuestas por cada nueva generación de historiadores" (Brush, 1974, pág. 1166). Se propone, así, no sólo que cada generación de historiadores vuelve a narrar cierta historia sobre la ciencia-objeto: a su vez se sostiene la novedosa idea de que la historia es, en cada una de esas generaciones, una reconstrucción, y no un relevamiento o una descripción de los hechos tal como sucedieron (como lo ordenaba la máxima rankeana).

La noción de que la historia de la ciencia se reconstruye con cada generación de historiadores fue parte de la perspectiva historiográfica de variados y disímiles autores en el campo de la psicología. Por mencionar algunos, el historiador Robert Watson, al explayarse sobre el rol y uso de la historia de la psicología en la formación de los psicólogos había reconocido que dicha historia, al responder a intereses concretos, y al pertenecer estos intereses a generaciones específicas, debía ser reescrita constantemente (Watson, 1966). Aunque implicaba un gran esfuerzo, la revisión de reconstrucciones históricas, y no su aceptación a priori, era para Watson la única forma procedimental con que contaba el historiador para asegurar un nivel aceptable de veracidad en su tarea. 
A su vez, Buss (1975) incorporaría la idea del carácter dinámico y generacional del conocimiento y de la cultura a partir de la teoría generacional del sociólogo del conocimiento Karl Mannheim (Mannheim, 1952), sosteniendo la dependencia generacional ya no sólo de las reconstrucciones históricas, sino del conocimiento psicológico general. Esta es, de hecho, una de las primeras inclusiones explícitas de teorías o perspectivas sociológicas para la explicación de alguna de las dimensiones de la ciencia psicológica.

La recomendación para el historiador acerca de mantener una actitud crítica y revisionista y de fundamentarse adecuadamente en fuentes primarias, sostenida inicialmente por los autores de la historia crítica (Danziger, 1979; Woodward, 1980), respondió en parte a la tendencia -de los historiadores y sociólogos foráneos a la disciplina 0 sin entrenamiento profesional- de aceptar acríticamente los relatos históricos heredados generacionalmente, y de usarlos como base de interpretaciones y ulteriores producciones historiográficas. El relato histórico heredado acríticamente a que aludía Danziger era, por supuesto, la Historia de la Psicología Experimental de Edwin Boring (Boring, 1950/1978), obra de inmensa erudición pero que sin embargo pretendía legitimar una imagen parcializada de la psicología y de su historia y respondía a los intereses intelectuales específicos de su autor (O'Donnell, 1979). La necesidad de una perspectiva crítica que Brush requiere en su texto, Danziger (1984) la establece como un requisito indispensable para una reconstrucción historiográfica crítica que, de forma reflexiva, aspire a algo más que constituir relatos celebratorios para iniciar a los académicos.

Brush reclama así y de forma explícita la ampliación del género de historia utilizado por los clásicos historiadores de la ciencia. En este sentido, identifica que ciertos historiadores de la ciencia -entre ellos el prolífico y pionero George Sarton-, aunque reconocen como legítimo el estudio de la historia y de la totalidad de los conocimientos pasados, llaman a no perder de vista que lo que ha sido dejado atrás por los científicos debe ser considerado necesariamente como perteneciente a los errores o fallos históricos. En este sentido, la historia sería sólo un cúmulo de instancias de aprendizaje fallidas. La historia clásica de la física, por ejemplo, enfatiza el rigor metodológico de autores como Galileo y Newton, en desmedro de otras variantes explicativas historiográficas y de otras teorías originalmente competidoras a los modelos teóricos propuestos por dichos autores.

Brush nota que esta actitud es diametralmente opuesta a lo que propone la nueva historia de la ciencia. Esta coloca en un segundo plano la importancia de la verificación experimental para el avance de la ciencia y eleva a objeto de estudio otros tipos de factores y estructuras (filosóficas, metafísicas o sociológicas) para el avance de la ciencia. Haciendo eco de la clásica sociología del conocimiento, y 
augurando el extremo al que la sociología del conocimiento científico (Bloor, 1976/1998) llevaría los planteos kuhnianos, Brush sostiene que el historiador, lejos de ser un documentador de la objetividad de los problemas en ciencia, "debe estar preparado para analizar los aspectos filosóficos, psicológicos y sociológicos del trabajo científico" (Brush, 1974, pág. 1166) a la hora de analizar desde una perspectiva histórica y epistemológica la prevalencia, aceptación, difusión o fracaso de teorías y enfoques científicos. En dos importantes sentidos amplía aquí Brush el campo de la historiografía de la ciencia: el objeto de esta historia ya no es el pasado definido como cúmulo de errores inevitables para alcanzar el presente, sino el pasado definido como conjunto de verdades relativas a su contexto histórico particular. En un segundo lugar, Brush da lugar en la historia de la ciencia a análisis filosóficos, psicológicos y sociológicos: análisis que durante la década siguiente registraron una gran proliferación, especialmente al debatir cuestiones epistemológicas en el cruce de la sociología, la filosofía y la historia (Medina, 1982; 1983)

Por lo anterior, Brush critica la concepción que él percibe como 'clásica' en Historia de la Ciencia, centrada en grandes hombres, sus descubrimientos y las sucesiones de dichos descubrimientos a partir de refutaciones experimentales. Aunque referido siempre a la ciencia física, no excluye de dentro el campo de la Historia de la Ciencia el estudio de cuestiones tales como las tradiciones o paradigmas de pensamiento. Así, dentro de la nueva historia de la ciencia, "los historiadores de la ciencia han comenzado a desenfatizar el contenido técnico de aquellas teorías que las hace significativas para la ciencia moderna" (Brush, 1974, pág. 1170), virando entonces hacia estudios de carácter más contextual.

Esta caracterización ampliada de la historia, especialmente en sus vertiente sociológica, reaviva hoy necesariamente los debates en torno a las problemáticas relaciones entre la sociología y la historia (Burke, 1994). Paralelo al examen crítico de Brush, comenzaba a sucederse una reapropiación de la historiografía deudora de Kuhn no sólo en la sociología (como es el caso del Programa Fuerte de Bloor) sino también en la propia Historiografía de la Ciencia. Uno de los representantes de la nueva historia, Steven Shapin, exponía en 1982 las cuantiosas investigaciones historiográficas que, cercanas a la sociología del conocimiento y de la ciencia, se arrogaban para sí el análisis tanto del contenido como de la difusión de las teorías científicas (Shapin, 1982); hecho este que en cierta medida reavivó la polémica del internalismo y externalismo en historia de la ciencia (Medina, 1983).

El debate entre el internalismo y el externalismo en la historia de la ciencia remite al lugar y poder explicativo de los factores científicos y extracientíficos en la dinámica de la ciencia y más precisamente en el conocimiento producido. El internalismo en Historia de la ciencia 
sostiene que el conocimiento científico producido obedece sólo a factores identificados como inherentes al ámbito de la ciencia: la argumentación lógica y la contrastación empírica de hipótesis. La sociología internalista de la ciencia (Merton, 1977), a su vez, limita su campo de estudio a las relaciones sociales de los científicos y sus normas sociales. En su dimensión normativa, el internalismo sostiene que

La ciencia avanzará por el camino adecuado no sólo cuando sea capaz de tomar decisiones correctas a partir de su partir de su propia lógica sino, además, cuando logre emanciparse de lo que no pertenece estrictamente a la caverna, a su juego de luces y sombras: las fuerzas sociales, políticas y económicas, la injerencia del poder, el debate sobre la utilización pragmática de los avances científicos, las polémicas sobre la moralidad, el modo en que los seres humanos van asumiendo históricamente la aparición de cada una de las novedades científicas. (Medina, 1983, pág. 55)

El externalismo, o la generalidad de sus múltiples variaciones, sostiene que es importante para un análisis de la producción del conocimiento científico el conocimiento y descripción de las incidencias que se dan sobre el campo académico desde los factores que le son externos, entre los cuales algunos son los citados por Medina. Una diferenciación entre externalistas extremos (los historiadores marxistas y los sociólogos relativistas que a partir de 1980 profundizaron las tesis de Bloor) y externalistas moderados (o sofisticados, que reformulan estos planteos relativistas) excede este trabajo; valga aquí la característica esencial de esta postura en historia y sociología de la ciencia que sostiene que los factores sociales, políticos, económicos, culturales e institucionales tienen un papel no accesorio en la producción, difusión y legitimación del conocimiento científico.

La propuesta de Brush (y de otros sociólogos e historiadores sociales de la ciencia) acerca de una historia orientada contextualmente puede verse consumada en la historiografía de la psicología, específicamente, en el enfoque de la historia social de corte críticomarxista (Wolf, 1978), en la historia sociológica de corte mannheimiano (Buss, 1975; Danziger, 1979) o en la historia social de corte profesionalista o institucional (Sokal, 1984; Vilanova, 1997), entre otros. Estos tres marcos (distintos pero que pueden agruparse dentro de la rúbrica de 'historia social' de la psicología) comparten en común la negativa a limitarse a reconstrucciones internas de la historia de la ciencia, reconociendo explícitamente la hipótesis de que los procesos de producción de conocimiento (especialmente aquellos en perspectiva histórica) no pueden comprenderse totalmente sin 
considerar el ambiente más amplio de la ciencia. Aunque distintos estudios enfatizan distintas esferas (como la cultural, la política, la económica, la institucional), todos estos géneros históricos coinciden en realizar contextualizaciones sociales de las teorías y modelos psicológicos; en ocasiones, consideran al contexto como recurso explicativo de la emergencia y difusión de la psicología (Ash, 1993).

No se registra, sin embargo, una proliferación tal de enfoques en lo que respecta a la articulación sociología-historia en el marco de perspectivas de enseñanza de la historia de la psicología. En cierto sentido, en la enseñanza se produjo un efecto inverso a los desarrollos investigativos: el viraje de la historia clásica (internalista) a la nueva historia (externalista) produjo "un aumento del distanciamiento entre las metas del historiador y las del docente de ciencia" (Brush, 1974, pág. 1170). La historia de la ciencia internalista, dada su lógica de análisis, necesariamente implica una reconstrucción circunscripta a aspectos teóricos y metodológicos de la ciencia con poca atención a factores externos; y aunque no es estrictamente necesario, sí es lógico esperar que dicha reconstrucción se centre en los aciertos y los descubrimientos sucesivos que hayan permitido, a lo largo de la historia de la ciencia, una acumulación de conocimientos, más que en las teorías erróneas u olvidadas. Por el contrario, la historia externa (fuertemente aleada con la sociología de la ciencia en algunas de sus variaciones) hará hincapié en el poder explicativo y efectivo de las instancias extra-científicas sobre la comunidad científica, sus prácticas y sus productos cognoscitivos. Esta historia es precisamente la que radicaliza la idea kuhniana de progreso cualitativo, fundamentando la dinámica científica no en la acumulación positiva de conocimientos fácticos sino a través de instancias esencialmente pragmáticas tales como las negociaciones retóricas, las revoluciones científicas, los intereses y conflictos de grupo y de clase, etcétera. La meta del historiador externo, en términos de Brush, ya no es resguardar la caverna, por tomar la metáfora de Medina, sino precisamente iluminarla y mostrar el irracionalismo propio (aunque no total) de las prácticas científicas. De aquí que la 'nueva historia de la ciencia' sea un mal ejemplo para los alumnos, y por tanto deba ser censurada. Así, es comprensible que la redefinición del género y foco de la Historiografía de la Ciencia no fuera adoptado por los docentes de ciencia contemporáneos a Brush. Podemos hallar, sin embargo, ciertas repercusiones en las fundamentaciones y propuestas de la historia de la psicología como curso de grado. En efecto, existen algunos pronunciamientos de autores internacionales a favor de una enseñanza de la historia (de la psicología) que presente a la ciencia como social y culturalmente contextuada (Benjamin, 2009; Bohan, 1990; Chamberlin, 2010; Robinson, 1979). Estas propuestas, en cierto sentido, se han apropiado críticamente del argumento de Brush, demostrando que 
puede impartirse una historia de la ciencia predominantemente reflexiva y autoconciente. A nivel regional, hallamos entre otras las argumentaciones del historiador argentino Alberto Vilanova (Vilanova, 2000) en torno a la enseñanza de una historia de corte social o sociológico, desarrolladas más adelante.

\section{La tensión entre la enseñanza de la Historia y el carácter del conocimiento histórico: whiggismo, presentismo y criticismo}

En el marco del conflicto entre la historia clásica y la historia en clave kuhniana plantea Brush uno de los dilemas más importantes para la posteridad de la disciplina histórica: en el ámbito investigativo, la aparente contradicción entre el historiador de la ciencia (revisionista) y el científico mismo (usualmente más conservador, en términos de Brush). En el ámbito propiamente formativo - la enseñanza universitaria de la historia -, la problemática relación entre la enseñanza de la ciencia (especialmente la enseñanza de la historia como curso separado) y la historia como cuerpo de conocimiento de carácter revisionista y crítico.

Respecto al dilema en el ámbito investigativo, Brush reconoce que existe una brecha necesaria entre el historiador profesional de la ciencia y el científico de la ciencia de que se trate: el primero dedica su tiempo a estudiar de forma coherente las teorías, personajes y condiciones socioculturales de la ciencia, mientras que el otro necesariamente se halla ocupado resolviendo los problemas diarios que demanda el avance del conocimiento. El ámbito del primero es el pasado (aunque en relación con el presente, dado que esto es inevitable); el ámbito del segundo es el laboratorio (entendido aquí de forma laxa, como cualquier espacio inmediato donde el científico realice su labor, sin referencia a la historia).

Esta diferencia fue percibida en psicología por el ya citado Ash (1983), como también por Kurt Danziger. En línea con Brush, Danziger argumentaba que los psicólogos, a través de sus prácticas, tienden a establecer 'objetos' psicológicos que, en tanto ligados a contextos y momentos históricos específicos, son eminentemente epocales y requieren de legitimación de contextos intra y extra científicos (Danziger, 1995). Respecto de la compleja relación entre el historiador y el científico, Danziger sostenía que

Al estar libres del corset del "whiggismo", [los historiadores de la ciencia] a menudo producen un conocimiento histórico intrínsecamente más valioso, pero deben pagar un precio: el aislamiento en relación a la comunidad de los científicos. El público al cual llegan los historiadores de la ciencia seguramente está formado por otros historiadores y no por 
científicos. De modo que la profesionalización y la creciente autonomía de la historia de la ciencia en realidad fortalecen la ideología de la científica según la cual historia y metodología se excluyen mutuamente. (Danziger, 1993, s.p)

El whiggismo al que hace referencia Danziger es el sesgo historiográfico que, en historia de la ciencia y de la psicología, ubica en el presente el estado de mayor conocimiento de la psicología, identificando el pasado - como ya se aludió en el apartado anterior como un una serie de instancias erróneas que implican, por tanto, estados de menor conocimiento (Lovett, 2006). La nueva historia de la psicología, a partir de retomar influencias específicas desde la historia kuhniana y post-kuhniana de la ciencia, precisamente se orienta en el sentido de abandonar el whiggismo y otros sesgos historiográficos característicos de la historia clásica, lineal y acumulativa. La nueva historia de la psicología tiene, como características historiográficas distintivas, una orientación esencialmente distinta de la historia clásica. En este sentido, se orienta por el historicismo, intentando retratar a los autores y teorías en los términos y contextos de sus propias épocas, minimizando la injerencia del presente (Furumoto, 1989).

Esta injerencia del presente implica, precisamente, otro juicio historiográfico denominado 'presentismo'. Tanto en su versión clásica como en su versión crítica (Buss, 1977), implica utilizar el conocimiento y valores disponibles en la actualidad para orientar preguntas, investigaciones y valoraciones en Historia de la ciencia. Definida así la nueva historia de la psicología, es claro el conflicto que traba con la historia clásica, en tanto que implica necesariamente una tendencia superadora de las carencias e ingenuidades de la segunda. Esta confrontación de intereses y objetivos en el ámbito investigativo tiene su traducción necesariamente en el área de la enseñanza, específicamente en la formación universitaria de grado. Las investigaciones del historiador, a la vez que no llegan a los teóricos básicos, tampoco suelen ser retomados por los propios docentes, encargados de la introducción de los universitarios en el ámbito científico y profesional.

Cuando el docente de ciencia presenta materiales históricos, debe hacerlo de una forma muy selectiva, dado que su propósito real debería ser enseñar teorías modernas y técnicas de una forma más efectiva; sólo puede tomar del pasado lo que tiene significación para el presente. El resultado puede ser una serie de fascinantes ( $y$ a menudo míticas) anécdotas, pero esto seguramente no es lo que el historiador entiende por historia. (Brush, 1974, pág. 1166).

Así, Brush sostiene que la identificación del docente con un historiador whig es de carácter necesario. El whiggismo es el cariz propio de las historias donde el conocimiento se muestra como un 
continuo lineal y progresivo y donde la actualidad científica representa el conocimiento verdadero, mientras que el pasado necesariamente queda relegado como un conjunto de teorías predominantemente erróneas (Danziger, 1993; Goodwin, 1997). En la visión whig de la historia se intersectan dos errores de juicio historiográficos: el justificacionismo o celebracionismo (puesto que se evalúa selectivamente a las personalidades y teorías del pasado en términos de lo que han colaborado para el estado actual de la ciencia, y usualmente en términos de aciertos y errores) y el presentismo (puesto que los criterios con que se evalúa el pasado de la ciencia provienen de los conocimientos, valores y teorías científicas contemporáneas).

Si consideramos que la historia, en el currículo universitario, persigue el fin de fundamentar o comprender -al menos indirectamente- el estado actual de la ciencia, entonces por cuestiones funcionales y programáticas (principalmente, la economía del tiempo), el docente enseñará como acertada la línea histórica que conduce hasta el presente. En este sentido, "uno podría decir que la historia whig es precisamente la que quiere el docente- él está interesado sólo en aquellos desarrollos anteriores que llevan a teorías y leyes establecidas en la actualidad" (Brush, 1974, pág. 1170). Aquellos contenidos históricos que no conduzcan de alguna forma al estado actual del área científica de que se trate no formará parte del currículo, o será incluida pero en calidad de error o distractor del progreso científico ( $y$, como sostiene Brush, es muy probable que simplemente no se enseñe). En otras palabras, el docente, según Brush, reproduce en la enseñanza la situación de incomunicación que él y Danziger ubican originariamente en el ámbito investigativo.

Respecto a las posibles resoluciones de estas situaciones ambiguas, y circunscribiéndonos ya a la cuestión de la Historia de la ciencia en la enseñanza y la formación de grado, debemos notar que Brush parece permanecer escéptico de una resolución favorable para los alumnos. Según Brush, es difícil imaginar un docente de ciencia que, al entrar en contacto con la nueva historia de la ciencia y aceptando los argumentos y revisiones que esta plantea, no desarrolle cierto escepticismo sobre la objetividad del conocimiento; escepticismo que, aunque deseable de ser transferido al alumno como estímulo al pensamiento crítico, necesariamente va en contra de los usos pedagógicos de la Historia -los cuales son ampliamente mayoritarios- Al ser estos usos los predominantes, es difícil concebir en un primer momento la existencia de un docente revisionista en un curso de Historia de la ciencia. Por esto, concluye Brush, es más plausible concebir que, simplemente, los avances historiografía e historia de la ciencia no alcanzarán a los docentes en sus actividades. En psicología, el aislamiento de los docentes y de los manuales respecto de los avances investigativos en historia de la psicología han sido 
ampliamente documentados (Ash, 1983; Bhatt \& Tonks, 2002; Fuchs \& Viney, 2002).

Esto, sin embargo, no significa que ningún docente sea capaz de resignar los fines adoctrinadores de la Historia de la Ciencia a favor de una actitud más crítica. El mismo Brush releva casos donde la nueva historia de la ciencia es, de hecho, incorporada al currículo. Y aunque la prospectiva de combinar materiales críticos con materiales clásicos ubica al docente de Historia en una posición extraña o paradójica, la situación no está signada por la imposibilidad. En este sentido sostiene que existen docentes que "quieren contrarrestar el dogmatismo de los manuales y transmitir una comprensión de la ciencia en tanto actividad que no puede ser divorciada de consideraciones metafísicas o estéticas" (Brush, 1974, pág. 1171).

Esto requiere que el docente -0 el curso- de historia no retraten a la historia en términos de linealidad hacia el presente, presentando varias tradiciones históricas en psicología, junto con controversias y resoluciones -no totalmente racionales- de algunos debates científicos. Y el resultado de tales actividades son, inevitablemente, los aspectos subversivos, tal como los denomina Brush, de la enseñanza de la historia. En este sentido, el alumno ya no es socializado en torno a reconstrucciones míticas de la ciencia a cuyo estudio se aboca, sino que es confrontado con una pluralidad de vías históricas, con un conjunto de alternativas posibles (ya no juzgadas como erróneas o verdaderas) y con una historiografía que sostiene que la corroboración de hipótesis a través de experimentos no es el único motor de la dinámica científica. Si la docencia whiggista es reemplazada por una docencia crítica en historia de la ciencia, permite capitalizar "el uso educacional de la disciplina [e] inculcar claridad y pensamiento crítico" (Brush, 1974, pág. 1171).

La cuestión sobre los efectos subversivos es de especial relevancia en el campo que nos convoca, puesto que a diferencia de las ciencias naturales - al menos las ciencias naturales de 1970, cuya modelización es objeto de las reflexiones de Brush-, la psicología consta de un largo historial de manifestaciones y atribuciones de crisis debido a la pluralidad de tradiciones teóricas en su interior (Sturm \& Mülberger, 2012). Así, a partir de los planteamientos de una historia policéntrica de la psicología (Brock, 2014) que muestra un nacimiento igualmente policéntrico de la psicología (Danziger, 1979), es difícil concebir una propuesta curricular de historia de la psicología que sólo presente una línea histórica y a la vez no sea excesivamente parcial 0 sesgada: dado que las tradiciones psicológicas del siglo XIX y XX han sido múltiples, y dado que en la actualidad no podemos hablar de una psicología unificada. Por tanto, el problema planteado por Brush se reformula: de la necesidad de enseñar ya no una sino varias reconstrucciones históricas alternativas (el desafío de la enseñanza de la historia de la física de 1970, por 
ejemplo) hacia la necesidad de delimitar, justificada y racionalmente, cuáles líneas o tradiciones históricas conformarán el contenido de la historia de la psicología como curso de grado, si su referente (la psicología) es una ciencia eminentemente pluralista o fragmentada.

Sin pretender zanjar la cuestión podemos decir, respecto al docente whiggista, que entre los historiadores de la psicología no se sostiene ya la creencia de que la enseñanza deba ser inherentemente presentista o celebracionista, tal como el propio Brush parece haber reconocido. Puede serlo, por supuesto. Pero los adelantos académicos, institucionales e investigativos de la historia de la psicología ya dejan poco margen para que los cursos se limiten a un solo enfoque, línea o perspectiva histórica (Vaughn-Blount, Rutherford, Baker, \& Johnson, 2009; Rutherford, 2013). Si el docente contemporáneo de historia es whiggista, presentista o celebracionista, lo será por elección o por defecto de formación, mas no por una necesidad supuesta respecto de su rol ocupacional.

Respecto a la estructura del curso de historia y de sus potenciales efectos subversivos, notamos cierto consenso en torno a la deseabilidad de un curso de grado de historia que presente al alumno cierta diversidad de alternativas teóricas en el campo científico de la psicología (Rosa, Huertas, \& Blanco, 1998; Dagenbach, 1999). Esto no impide el centramiento o definición del curso de historia en torno a algunos núcleos problemáticos delimitados. Sí, claramente, exige una exposición relativamente ordenada de múltiples propuestas históricas en psicología, cuyo escamoteo sería pernicioso para el desarrollo de su juicio crítico y para su formación académica y profesional (Vilanova, 1996; 1997; Baker, 2002).

Es precisamente a través de la enseñanza y aprendizaje de la historia que, según ciertos autores (Robinson, 1979; Goodwin, 1997; Benjamin, 2009), se pretende desarrollar competencias críticas, tanto teóricas como prácticas, respecto del desempeño en psicología. Las asignaturas de corte histórico serían el lugar idóneo para enseñar acerca de los problemas nucleares que encierran las actitudes presentistas o justificacionistas, permitiendo así y entre otras cosas una comprensión cabal no sólo del campo historiográfico de la psicología sino también de las teorías y metodologías propias de la ciencia (Rosa et al., 1998).

La cuestión de la crítica misma ocupa un lugar central en las referencias contemporáneas dentro de los estudios históricos (Ash, 1983; Danziger, 1993; Benjamin, 2009). Aunque para ciertos autores la idea misma de un curso (institucionalizado) de historia de la psicología dentro de una carrera universitaria que permita el desarrollo del pensamiento crítico es un oxímoron (Danziger, 1993), retomamos aquí posturas más optimistas en torno a la cuestión de la enseñanza y sus posibilidades (Vilanova, 1996; Benjamin, 2009). Estas posturas sostienen que, en primer lugar, la historia de la 
psicología -enseñada desde una perspectiva pluralista y a partir de modelos historiográficos actualizados- es susceptible de desarrollar el pensamiento crítico del alumno, mostrándole la no necesariedad del conocimiento psicológico (su historicidad) y permitiéndole así el replanteamiento reflexivo de lo que en otro caso sería un dogma preestablecido. Una visión crítica, tanto en la actividad investigativa de la historia como en su enseñanza de grado, implica disposiciones y competencias idénticas: en términos de Benjamin (2009), la crítica implica reflexionar acerca de lo que a uno se le pida que crea, implica evaluar la evidencia que sustenta el pedido de creencia, implica evaluar las explicaciones alternativas que uno puede generar sobre el fenómeno objeto de la creencia, e implica reflexionar acerca de las evidencias que deberían buscarse para refutar las alternativas.

En segundo lugar, estas posturas sostienen que lo que diferencia al docente whiggista del docente capaz de transmitir esta actitud crítica y reflexiva es la adopción, a su vez y por su parte, de una actitud crítica respecto de la disciplina, del conocimiento que esta produce, y de su propia labor docente, lo cual según los autores citados y según experiencias y cursos históricos concretos es factible de realizarse. Esta actitud crítica que se exige al docente de historia de la psicología puede entenderse en, al menos, dos sentidos. Primero, como cierto escepticismo actitudinal saludable, moderado, informado y reflexivo respecto de los mitos, reconstrucciones manualizadas y enfoques historiográficos excesivamente limitados presentes en el ámbito (Brush, 1974, pág. 1170; Vaughn-Blount et al., 2009). Este escepticismo permitiría una aproximación y análisis objetivo tanto de las fuentes primarias como de las fuentes a ser impartidas en los propios cursos de historia. Segundo la actitud crítica puede concretizarse en el esfuerzo por contextualizar social y culturalmente las tradiciones históricas que forman parte del currículo y del curso de historia de que se trate. En términos de Vilanova (2000),

Una historia acrítica [...] puede ser yugulada, en buena medida, teniendo conciencia de que los conceptos psicológicos son producidos por agentes sociales. [...] Una historia crítica de la Psicología debe ser una historia histórica, en el sentido de ser sociológica, procurar el suelo social de los conceptos, preocuparse por los productores de conocimiento como agentes representantes de grupos de interés [...] Una historia social, sociológica, con sus raíces profundamente hundidas en los problemas socioeconómicos y políticos de la región y de la época de la que se trate. (Vilanova, 2000, pág. 146)

En este sentido, una enseñanza será crítica -en el sentido ya expuesto- en la medida en que contextualice a los autores, teorías y sistemas psicológicos en esferas más amplias, de las que se supone 
que emergen y toman sentido. Esta contextualización, abordable de múltiples formas concretas, debería según el planteo de Vilanova exponer las raíces económicas, políticas, sociales, culturales y profesionales de las teorías psicológicas. Este es, como se esbozó antes, el argumento principal tanto de la sociología del conocimiento clásica (Mannheim, 1952) como de la sociología del conocimiento psicológico (Buss, 1975). La criticidad aquí radicaría en que la contextualización social habilitaría una comprensión más cabal de los contenidos históricos de la ciencia, de las teorías que la componen y de las problemáticas para las que dichas teorías son respuestas, a la vez que situarían en perspectiva histórica (no presentista) los elementos que componen el pasado disciplinar. En términos de Benjamin (2009), quizá el más sistemático pedagogo de la historia de la psicología, sostiene que

La psicología no existe en un vacío. Trabajamos en un campo que está influenciado en gran medida por lo que sucede en nuestro alrededor. La geografía, la política, el arte, la religión, la cultura, la literatura, y otras, son las fuerzas que moldean nuestra disciplina. Comprender la psicología significa que seas capaz de verla como parte de su contexto. Es el contexto el que enriquece la interpretación y la comprensión. (p. 180. Énfasis agregado).

\section{Discusión}

Aunque la célebre y pionera comunicación de Brush se movía dentro de las cuadrículas de la historia de las ciencias naturales (específicamente las físicas), hemos visto que las temáticas planteadas por su exposición fueron luego cuestiones con cuerpo propio dentro de la historia de la psicología, espacio en el que se recepcionaron en calidad de desafío a ser superado en tanto insignia de la historia pedagógica.

En primera instancia, esta recepción se da a partir de dos cuestiones eminentemente teóricas 0 historiográficas: las reconstrucciones históricas como emergentes generacionales y siempre cambiantes, y la ampliación del género histórico que sirve de base a la historia de la ciencia, y la consiguiente ampliación de la tarea propuesta para el historiador. En este sentido, se muestra que desde el surgimiento de lo que conocemos como nueva historia, o historia crítica, de la psicología, los historiadores han tenido mayor sensibilidad respecto del carácter problemático de la historia (dado que cada relato histórico es una reconstrucción interesada) y de la necesidad de trascender el género de historia política aplicada a la historia de la psicología (que en sus exponentes clásicos se traducía en una historia 
individualista, personalista y realista ingenua). En este espacio es que se reiteran, de forma particular en historia de la psicología, los debates contemporáneos a Brush sobre la historia interna y la historia externa de la ciencia, en gran medida iniciados por los debates en torno a Kuhn. A estos debates se agregó pronto la sociología de la ciencia, impulsando aún más la imagen de que una historia interna era una reconstrucción parcial e incompleta del pasado científico. Ante estos debates, la nueva historia de la psicología ha optado generalmente por orientarse por las vertientes externalistas, especialmente las que defienden el valor explicativo e interpretativo de los factores sociales sobre la producción del conocimiento científico.

De especial interés, sin embargo, son las implicaciones que para la enseñanza de la historia de la psicología tuvieron las problemáticas para las cuales los argumentos de Brush pretendían ser reflexiones y respuestas: la visión de la historia clásica de la ciencia, predominantemente presentista y whiggista. En efecto, según Brush y de cara a toda una tradición de enseñanza de historia de la ciencia, era imposible por supuesta necesidad una enseñanza de la historia de la ciencia que no considerase al pasado como un cúmulo de errores y al presente como el estado de mayor conocimiento desde el cual se investigase, evaluase y valorizase la historia, tanto por la propia estructura universitaria como por la formación y convicciones de los instructores.

Hemos destacado la importancia de las temáticas del whiggismo, del presentismo y justificacionismo, y hemos relevado las posturas ante la problemática que representa una enseñanza crítica de la historia en un marco universitario que se supone pretende, predominantemente, socializar a iniciados. Aunque los historiadores y docentes que integran el campo de la historia de la psicología reconocen que no son peligros insalvables, es cierto que nada dentro de la institución universitaria -salvo cierto consenso académico, que a su vez es más propio del ámbito anglosajón- obliga a los cursos de historia a adoptar posturas que superen estos sesgos historiográficos. Aunque el docente no necesariamente deba conducirse de forma whiggista, tal como sostenía Brush, lo cierto es que no existen condiciones infraestructurales u objetivas que se lo impidan, y autores como Danziger (1993) no se han demostrado demasiado optimistas respecto de docentes y cursos de historia que no apunten exclusivamente a socializar a los iniciados a través de relatos históricos distorsionados y sobresimplificados.

Lo mismo puede decirse de algunos de los efectos deseados de la enseñanza de la historia de la psicología, especialmente en lo que respecta al desarrollo del pensamiento crítico: aunque las líneas curriculares aludan implícitamente al pluralismo, los cursos de historia bien pueden presentarse a partir de enfoques 
(historiográficos 0 curriculares) excesivamente limitados 0 desfasados, o pueden presentar una aparente pluralidad tamizada en la práctica por perspectivas epistemológicas o historiográficas parcializadas e interesadas. Lo que parece subyacer a los planteos tanto de Brush como de Danziger y de pedagogos regionales como Vilanova, es la convicción de que la disposición natural de una asignatura histórica no se orienta necesariamente a la criticidad, sino a su contrario -el adoctrinamiento-. Esto sería consecuencia de, entre otras cosas, la finalidad misma de la enseñanza o formación en ciencia clásicamente concebida -que no debe reparar en 'errores' del pasado- y de la desconexión entre la investigación en historia y su incorporación como contenido de formación de grado (es decir, del cisma entre los adelantos investigativos y su incorporación en las asignaturas históricas). Los autores coinciden que, para evitar las consecuencias negativas que se siguen de esto, se requiere un esfuerzo explícito y deliberado.

Es en el sentido de subsanar algunas de las carencias de la enseñanza de la historia de la psicología ya aludidas, y de promover no el irracionalismo sino los desenlaces deseables y productivos de aquellos efectos subversivos citados por Stephen Brush hace cuatro décadas, que retomamos explícitamente la alusión (o exhortación) a los docentes-historiadores acerca del desarrollo de un sano escepticismo crítico, como una de las formas concretas de entre las muchas posibles en que puede materializarse el esfuerzo explícito arriba referido. En vínculo con esto, y también a partir de Brush y de los nuevos enfoques históricos que surgieron contemporáneos a él, se concluye que la adopción de un marco de historia social o sociológica aplicada a la psicología es una de las soluciones meditadas y posibles ante el escenario que se materializa en las aulas y foros de los psicólogos desde hace varias décadas -donde el caso del psicólogo rioplatense es un caso ejemplar-: el de una lucha entre un irracionalismo relativista, ciego, contra un cientificismo estéril, no menos miope. No es ocioso aquí reconocer que esta lucha entre relativismo y cientificismo ha sido, ella misma, el eje de los debates más importantes en historia, filosofía y sociología de la ciencia en el siglo pasado (Medina, 1983; Shapin, 1982). De lo que se trata es de emular en los contextos curriculares y formativos específicos la civilización de los debates académicos en los campos referidos.

Aunque la historia de la psicología en tanto disciplina no se reduce a su faz propedéutica, es en la forma de cursos de grado y de contenidos curriculares que se presenta predominantemente en el paisaje académico en lo que respecta a la formación universitaria y al entrenamiento profesional. Probablemente sea a través de la actitud crítica y reflexiva descrita en el trabajo -insignia de la nueva historia desde sus inicios- que se logre avanzar en la resolución de la paradoja, de la que Brush hace 40 años analizó algunas aristas, en 
torno a la distancia entre la Historia de la psicología y la psicología como ciencia, y entre la Historia de la psicología como disciplina en sí misma y la Enseñanza de la Historia de la Psicología a nivel universitario.

\section{Referencias}

Ash, M. (1983). The Self-Presentation of a Discipline: History of Psychology In The United States Between Pedagogy and Scholarship. En L. Graham, W. Lepenies, \& P. Weingart (Eds.). Functions and Uses of Disciplinary Histories (pp. 143-189). Dordretch: Reidel.

Ash, M. (1993). Rhetoric, Society and the Historiography of Psychology. En H. Rappard, P. Van Strien, L. Mos, \& W. Baker (Eds), Annals of Theoretical Psychology, Vol. 8 (pp. 49-57). Nueva York: Plenum Press.

Baker, D. (2002). Historical Understanding and Teaching in Professional Psychology. History of Psychology, 5(3), 219-223. doi: 10.1037/1093-4510.5.3.219

Benjamin, L. (2009). History of Psychology as a Capstone Course. En D. Dunn, B. Beins, M. McCarthy, \& W. G. Hill(Eds.). Best Practices for Teaching Beginnings and Endings in the Psychology Major (pp. 171-186). Oxford, Inglaterra: Oxford University Press.

Bhatt, G., \& Tonks, R. (2002). What lies in the future of teaching the history of psychology? History and Philosophy of Psychology Bulletin, 14(1), 2-9.

Bloor, D. (1976/1998). Conocimiento e Imaginario Social. Barcelona: Gedisa.

Bohan, J. (1990). Social constructionism and contextual history: An expanded approach to the History of Psychology. Teaching of Psychology, 17, 82-89.

Boring, E. (1950/1978). Historia de la psicología experimental. México D.F., México: Trillas.

Brock, A. (2014). What is a polycentric history of psychology? Estudos e Pesquisas em Psicología, 14(2), 646-659.

Brush, S. (1974). Should the History of Science Be Rated X? Science, 183(4130), 1164-1172.

Burke, P. (1994). Sociología e Historia. Buenos Aires: Alianza.

Buss, A. (1975). The Emerging Field of the Sociology of Psychological Knowledge. American Psychologist, 30(10), 988-1002. doi: 10.1037/0003-066X.30.10.988

Buss, A. (1977). In defense of a critical-presentist historiography: The fact-theory relationship and Marx's epistemology. Journal of the History of the Behavioral Sciences, 13(3), 252-260. doi: 
10.1002/1520-6696(197707)13:3<252::AID-

JHBS2300130305>3.0.CO;2-0

Chamberlin, J. (2010). Don't know much about history. Monitor on Psychology, 41(2), 44.

Dagenbach, D. (1999). Some Thoughts on Teaching a Pluralistic History in the History and Systems of Psychology Course. Teaching of Psychology, 26(1), 22-28. doi: 10.1207/s15328023top2601_4

Danziger, K. (1979). The Social Origins of Modern Psychology. In A. Buss (Ed.), Psychology in Social Context (pp. 27-45). Nueva York: Irvington.

Danziger, K. (1984). Towards a Conceptual Framework for a Critical History of Psychology. Revista de Historia de la Psicología, 5(1), 99-107.

Danziger, K. (1993). Tres desafíos para la Historia de la Psicología. Conferencia invitada expuesta a la División 26 en la Convención anual número 101 de la American Psychological Association [Trad.: Alejandro Dagfal]. Toronto. Recuperado em 20 de Agosto de 2014, de : http://www.elseminario.com.ar/biblioteca/Danziger_Desafios_hi storia_psicologia.htm

Danziger, K. (1995). The Production of Psychological Knowledge by Experts. Cuadernos Argentinos de la Historia de la Psicología, $1(1 / 2), 259-273$.

Fuchs, A., \& Viney, W. (2002). The Course in the History of Psychology: Present status and Future concerns. History of Psychology, 5(1), 3-15. doi: 10.1037/1093-4510.5.1.3

Furumoto, L. (1989). The new history of psychology. En I. Cohen (Ed.). The G. Stanley Hall Lecture Series (Vol. 9, pp. 5-34). Washington, D.C: APA.

Gooday, G., Lynch, J., Wilson, K., \& Barsky, C. (2008). Does Science Education Need the History of Science? Isis, 99, 322-330. doi: 0019-9902/2008/9902-0005

Goodwin, J. (1997). The vital role of Psychology's history in Introductory Courses: An Interview with Ludy T. Benjamin, Jr. Teaching of Psychology, 24(3), 218-221. doi: 10.1207/s15328023top2403_20

Klappenbach, H. (2000). Historia de la historiografía de la psicología. En J. C. Ríos, R. Ruiz, J. C. Stagnaro, \& P. Weissman (Comps.). Psiquiatría, Psicología y Psicoanálisis: Historia y Memoria (pp. 238-268). Buenos Aires: Polemos.

Lovett, B. (2006). The New History of Psychology: A review and critique. History of Psychology, 9(1), 17-37.

Mannheim, K. (1952). The Problem of Generations. En K. Mannheim \& P. Kecskemeti (Ed.). Essays on the Sociology of Knowledge (pp. 276-323). Londres: Routledge \& Kegan Paul. 
Medina, E. (1982). Teorías y Orientaciones de la sociología de la Ciencia. Revista Española de Investigaciones Sociológicas, 20, 7-58.

Medina, E. (1983). La Polémica Internalismo Externalismo en la Historia de la Ciencia. Revista Española de Investigaciones Sociológicas, 23, 53-75.

Merton, R. (1977). La Estructura Normativa de la Ciencia. En R. Merton(Ed.). La Sociología de la Ciencia 2 (pp. 309-370). Madrid, España: Alianza.

O'Donnell, J. (1979). The crisis of experimentalism in the 1920s. Edwin Garrigues Boring's and his uses of history. American Psychologyst, 34 (4), 289-295. doi: 10.1037/0003066X.34.4.289

Robinson, D. (1979). The History of Psychology and the Ends of Instruction. Teaching of Psychology, 6(1), 4-6. doi: $10.1207 / \mathrm{s} 15328023$ top0601_2

Rosa, A., Huertas, J. A., \& Blanco, F. (1998). Haciendo historia para el futuro de la psicología. Anuario de Psicología, 29(1), 73-87.

Rutherford, A. (2013). Teaching Diversity: What Can History Offer? History of Psychology, 16, 1-5. doi: 10.1037/a0034368

Shapin, S. (1982). History of Science and Its Sociological Reconstructions. History of Science, 20 (3), 157-211.

Sokal, M. (1984). James McKeen Cattell and American Psychology in the 1920s. En J. Brozek (Ed.). Explorations in the History of Psychology in the United States (pp. 273-323). Nueva Jersey: Associated University Presses.

Sturm, T., \& Mülberger, A. (2012). Crisis discussions in Psychology New historical and philosophical perspectives. Studies in History and Philosophy of Biological and Biomedical Sciences, 43, 425433. doi: $10.1016 / j$.shpsc.2011.11.001

Vaughn-Blount, K., Rutherford, A., Baker, D., \& Johnson, D. (2009). History's Mysteries Demystified: Becoming a PsychologistHistorian. American Journal of Psychology, 122 (1), 117-129.

Vilanova, A. (1996). Enseñanza de la Psicología: Historia y problemas fundamentales. Cuadernos Argentinos de Historia de la Psicología, 2(1/2), 199-210.

Vilanova, A. (1997). La Historia de la Psicología y su sentido curricular. Clepios, 3(2), 18-23.

Vilanova, A. (2000). Diez Problemas en la Historia de la Psicología como Curso de Grado. In C. J. Ríos, R. Ruiz, J. C. Stagnaro, \& P. Weissmann (Comps.). Psiquiatría, Psicología y Psicoanálisis: Historia y Memoria (pp. 143-154). Buenos Aires: Polemos.

Watson, R. (1960). The History of Psychology: A Neglected Area. American Psychologist, 15(4), 251-255. doi: 10.1037/h0044284

Watson, R. (1966). The Role and Use of History in the Psychology Curriculum. Journal of the History of the Behavioral Sciences, 
2(1), 64-69. doi: 10.1002/1520-6696(196601)2:1<64::AIDJHBS2300020108>3.0.CO;2-U

Weimer, W. (1974). The History of Psychology and Its Retrieval from Historiography: I. The Problematic Nature of History. Science Studies, 4(3), 235-258. doi: 10.1177/030631277400400403

Wolf, F. (1978). Marxian approaches to the history of psychology. Journal of the History of the Behavioral Sciences, 14, 122. doi: 10.1002/1520-6696(197804)14:2<122::AIDJHBS2300140204>3.0.CO;2-B

Woodward, W. (1980). Toward a Critical Historiography of Psychology. En J. Brozek, \& L. Pongratz (Eds.), Historiography of Modern Psychology (pp. 29-70). Toronto: Hogrefe.

\author{
Endereço para correspondencia \\ Catriel Fierro \\ Universidad Nacional de Mar del Plata \\ Facultad de Psicología \\ Deán Funes 3250, Cuerpo V Nivel III, Buenos Aires, BA, Argentina \\ Endereço eletrônico: catriel.fierro@gmail.com
}

Recebido em: 25/09/2014

Reformulado em: 19/11/2014

Aceito para publicação em: 20/11/2014

\title{
Notas
}

* Licenciando en Psicología. Becario de Investigación por la Universidad Nacional de Mar del Plata. Grupo de Investigación "Historia, Enseñanza y Profesionalización de la Psicología en el Cono Sur". Ayudante Estudiante en cátedra "Historia Social de la Psicología" 